OPEN ACCESS

Citation: Marciano C. (2021) Zero Waste. Gestione dei rifiuti e trasformazioni sociali, in «Cambio. Rivista sulle trasformazioni sociali», Vol. 11, n. 21: 149-161. doi: 10.36253/cambio-10632

Copyright: ( $) 2021$ Marciano C. This is an open access, peer-reviewed article published by Firenze University Press (http://www.fupress.com/cambio) and distributed under the terms of the Creative Commons Attribution License, which permits unrestricted use, distribution, and reproduction in any medium, provided the original author and source are credited.

Data Availability Statement: All relevant data are within the paper and its Supporting Information files.

Competing Interests: The Author(s) declare(s) no conflict of interest.
Open Essays and Researches

\section{Zero Waste. Gestione dei rifiuti e trasformazioni sociali}

\author{
Claudio Marciano \\ Università di Torino \\ claudio.marciano@unito.it
}

\begin{abstract}
The aim of the article is to analyse the social transformations produced and observed in the field of waste management. In particular, the focus is on the «Zero Waste» strategy, which has been proposing for at least two decades at a global level an alternative model of waste governance to the one centred on incinerators and landfills. The article proposes a theoretical framework to describe the genesis of «Zero Waste» as a discursive practice, proposing to observe jointly its characteristic symbolic, technological and organisational elements. In reporting the results of an ethnographic research conducted on the case of «Zero Waste» in Italy, the article also focuses on the processes of knowledge exchange between social movements, local administrations, universities and municipalised companies, the conjunctions between ecological movements and movements for the commons, the start of processes of remunicipalisation of local public services starting from a new strategy of legitimation of the public vs. the private, and the progressive institutionalisation of political ecology in the European and local policy arena.
\end{abstract}

Keywords: Zero Waste, environmental conflicts, waste treatment, socio-technical infrastructures, discursive practices, Environmental sociology.

La gestione dei rifiuti è un tema di rilevanza sociale e politica di portata glocale.

Circa 12 dei 17 SDG delle Nazioni Unite per il 2030 possono essere ricondotti ad aspetti relativi alla gestione dei rifiuti, in particolare al suo impatto per la sostenibilità e la qualità della vita nelle città (Rodić e Wilson 2017). Le direttive che la Commissione Europea ha finora approvato sul tema dell'economia circolare sono quasi interamente dedicate all'incremento delle raccolte differenziate e alla regolazione di un mercato delle materie prime secondarie (Marciano 2017). In Italia, il settore dei rifiuti genera un valore aggiunto di circa 12 miliardi di euro con il coinvolgimento di oltre 100mila addetti (Utilitalia 2020). A livello di governance locale, la gestione dei rifiuti è l'ambito dove i Comuni in forma singola o associata, possiedo- 
no ancora una normatività residuale (Mazzette e Sgroi 2007), potendo decidere in autonomia strategia e modello organizzativo del servizio.

La gestione dei rifiuti è, tuttavia, anche un campo sociale contrassegnato da interessi e visioni divergenti, dove si confrontano idee, tecnologie e modelli organizzativi tra loro alternativi. Per tale ragione, e per l'ampiezza delle trasformazioni sociali che si innestano o trovano in questo ambito tematico terreno di manifestazione, l'uso di alcuni strumenti dell'analisi sociologica può contribuire ad una loro maggiore comprensione.

L'articolo ha come oggetto la strategia «Zero Waste» (ZW), che si pone l'obiettivo di recuperare interamente il valore presente negli scarti, di ridurre radicalmente la quantità di rifiuti prodotti nelle città e di estinguere le soluzioni take-make-waste (TMW) fondate sull'uso di inceneritori e discariche (Connet 2013; Mazzarella et alii 2016; Romano et alii 2020). Adottando un'interpretazione pragmatica del concetto foucaultiano di «pratica discorsiva» (Bacchi e Bonham 2014), l'articolo propone di riconoscere in ZW un set di pratiche costituito da norme e valori incorporate in tecnologie e dispositivi organizzativi, la cui funzione è istituzionalizzare un modello di gestione dei rifiuti solidi urbani e, con esso, una visione del mondo, alternativa a quella dominante.

L'articolo ha tre obiettivi principali: i) descrivere le idee e i concetti caratteristici di «Zero Waste» adottando una prospettiva genealogica, pertanto analizzando il modo in cui il discorso è emerso e si è affermato in alternativa al paradigma TMW; ii) ricostruire gli eventi, gli attori e i processi politici più significativi che hanno portato all'affermazione del discorso di ZW in Italia; iii) mettere a fuoco alcuni fenomeni di mutamento sociale di cui è traccia e causa, dalla configurazione di nuove modalità di collaborazione tra attori sociali all'attivazione di processi di rimunicipalizzazione e di recupero della normatività da parte delle autorità politiche locali.

A un'analisi documentale e alla ricostruzione di un framework teorico interpretativo, l'articolo ha aggiunto come fonte un'indagine empirica condotta tramite la realizzazione di 15 interviste semi-strutturate con osservatori privilegiati e/o protagonisti dell'esperienza «Zero Waste» in Italia. L'obiettivo dell'indagine è stato quello di approfondire le ipotesi interpretative emerse durante la fase di analisi della letteratura e in particolare, di mettere a fuoco i processi generativi e diffusivi di ZW nel contesto italiano. Sono stati intervistati, in particolare direttori e amministratori unici di aziende che hanno adottato la strategia ZW nei propri ambiti di riferimento e che risultano essere, anche a livello pubblico, tra i principali promotori della visione; amministratori locali e attivisti che si sono distinti nel promuovere ZW nel proprio territorio ed in Italia; ricercatori e professionisti che hanno sviluppato in campo tecnologico, comunicativo e amministrativo soluzioni innovative per diffondere ZW nelle pratiche gestionali degli enti locali. La ricerca è stata svolta tra aprile e giugno del 2020, e le interviste si sono svolte esclusivamente tramite piattaforma online. La griglia utilizzata ha messo a fuoco tre diverse dimensioni: l'esperienza diretta dell'intervistato in relazione alla nascita di ZW in Italia; gli interscambi di conoscenza esplicita o tacita a cui ha partecipato per diffonderne la visione; i punti di forza e debolezza delle soluzioni prospettate da ZW rispetto al proprio contesto operativo o esperienziale.

L'articolo è strutturato nelle seguenti sezioni:

- La prima è dedicata alla messa a fuoco della letteratura scientifica e in particolare quella sociologica, che si è occupata di inquadrare se non ZW alcuni fenomeni legati alla sua emersione e affermazione;

- La seconda descrive le caratteristiche di ZW come pratica discorsiva mettendo a confronto gli apparati simbolici e materiali che ne caratterizzano l'identità rispetto al sistema TMW;

- La terza ricostruisce la genesi del movimento in Italia, mettendo a fuoco alcuni eventi che hanno favorito la diffusione di ZW in decine di contesti territoriali e l'interscambio di conoscenza tra diversi attori sociali;

- La quarta, infine, discute delle trasformazioni sociali che sono ravvisabili nell'esperienza di ZW in Italia.

\section{1. «ZERO WASTE»: DEFINIZIONI E RICERCHE.}

Nell'ambito della sociologia dell'ambiente e del territorio, si sono sviluppati negli ultimi venti anni almeno tre diversi filoni di ricerca sulla gestione dei rifiuti che, pur senza fare esplicito riferimento al fenomeno ZW, hanno messo a fuoco dei processi sociali che lo caratterizzano o hanno posto nel tempo le condizioni per la sua emersione. 
Il primo filone si è raccolto attorno all'analisi e descrizione dei processi di engagement della società civile o dell'expertise scientifica e professionale. In particolare, si sono approfondite le dinamiche di potere che si innestano tra attori sociali a diverso titolo coinvolti nei processi di conversione dei sistemi di raccolta o di realizzazione di infrastrutture di smaltimento. In questi studi, emerge il protagonismo delle reti civiche, delle associazioni ambientaliste e di volontariato, nella coprogettazione dei servizi (Osti 2002), specie nelle esperienze di coinvolgimento della cittadinanza in quartieri ad alto rischio sociale, dove ZW ha consolidato nuovi processi di auto-rappresentazione e ha dato voce alle diseguaglianze di riconoscimento (Martone 2016). Alcuni studi, analizzando sul campo l'applicazione dei sistemi di raccolta differenziata porta a porta in contesti urbani sfidanti come quello di Napoli, hanno messo a fuoco i fattori che tendono ad abilitare o meno un comportamento partecipativo e responsabile della popolazione (Zaccaria et alii 2012). A questo filone si possono associare anche gli studi, in particolare quelli di Pellizzoni (2011) sull'ingaggio delle expertise scientifiche nella valutazione e legittimazione degli impianti di trattamento dei rifiuti, e sulle conseguenze sociali e culturali dei processi di politicizzazione del sapere: in ZW il ruolo dei ricercatori è fondamentale, sia in termini di elaborazione delle pratiche discorsive, sia di legittimazione verso le autorità politiche e di diffusione.

Il secondo filone di studio si è dedicato all'analisi della struttura e delle dinamiche dei conflitti ambientali. Il contributo maggiormente rilevante di questi studi, ai fini della comprensione di ZW, è relativo all'analisi dei processi di mobilitazione dal basso e alle strategie argomentative nei conflitti. Le vertenze territoriali contro l'insediamento di inceneritori e discariche, o in occasione di emergenze rifiuti, sono state la culla di ZW perché hanno facilitato l'incontro tra amministratori locali, attivisti grassroots $^{1}$ o associazioni ambientaliste, scienziati e ricercatori e hanno offerto l'occasione di proporre un contro-modello rispetto a quello tradizionale. Sebbene molto diversi per le condizioni contestuali di partenza, tali processi si sono verificati in quasi tutti i casi studiati, documentati ad Acerra (Martone 2012; Avallone 2014), Trento (Magnani 2011), Torino (Tipaldo 2013; Crivello 2016), Parma (Pellegrino 2012).

Un terzo filone di studi ha analizzato il rapporto tra gestione dei rifiuti e strategie di governance dei servizi pubblici locali. Si tratta di un aspetto chiave che consente di analizzare in parallelo la diffusione della pratica discorsiva ZW e di quella relativa ai commons e alle rimunicipalizzazioni (Mattei 2018; Clifton et alii 2019). Su questo tema, Minervini $(2016 ; 2020)$, ha legato la gestione dei rifiuti al più ampio paradigma dell'economia fondamentale (Barbera e Jones 2020), mettendo in risalto il peculiare posizionamento delle aziende pubbliche in Italia - tra finalità pubblica e governance interna privatistica - e le implicazioni dei processi di quotazione in borsa e di finanziarizzazione del capitale sociale per il controllo da parte di cittadini e governi locali. La correlazione tra ownership pubblica e strategie di gestione dei servizi in ottica ZW è stata inoltre indagata in diversi lavori interdisciplinari a base empirica e comparativa (Romano et alii 2020; 2021), dove emerge il ruolo di ZW come mission tesa a ri-legittimare un ritorno alla gestione pubblica dei servizi pubblici locali.

Sebbene ognuna di queste ricerche mostri un aspetto di ZW nessuna di esse lo ha osservato frontalmente, come fenomeno in sé, né si è posto il tema di comprendere le ragioni della sua genesi e progressiva affermazione. La proposta del prossimo paragrafo è quella di avviare questo tentativo osservando come ZW sia emersa a partire dai limiti e dalle contraddizioni del sistema TMW, e si sia consolidata come una visione organica quando alcune realtà locali l'hanno messa in pratica.

\section{2. «TAKE MAKE WASTE»VS «ZERO WASTE»}

La gestione dei rifuti ha una sua storia, costituita da idee, tecnologie, valori, che si sono incorporati in modelli di governance del ciclo industriale.

\footnotetext{
${ }^{1}$ Il termine «grassroots» può essere tradotto in italiano con l'espressione «radici dell'erba». È un concetto utilizzato dalla sociologia dell'ambiente e del territorio per qualificare le esperienze di azione collettiva a livello locale condotte da comitati di base, organizzazioni spesso non strutturate né burocratizzate, e con una finalità istanziale, a volte limitata ad un singolo territorio (Strassoldo 1993).
} 
Nel modello TMW il ruolo di principale cleaner nel processo produttivo è ricoperto dalla discarica e/o dall'impianto di incenerimento. Il valore del rifiuto è prioritariamente economico, e viene calcolato con una formula in cui si sottraggono, ai costi per lo smaltimento, i rendimenti potenziali del recupero (Massarutto 2011) 2.

Lo smaltimento in discarica si è affermato nei paesi a capitalismo avanzato a partire dagli anni Sessanta, e si è configurato come una soluzione a basso costo per l'assorbimento delle prime grandi quantità di rifiuti solidi urbani (Pietzsch et alii 2017). Con la progressione esponenziale nella quantità di beni consumata, e anche con il mutamento nella qualità dei rifiuti da smaltire (più plastiche e rifiuti elettronici), la discarica è divenuta una soluzione meno redditizia e socialmente accettata rispetto a quella dell'incenerimento, che garantisce anche la valorizzazione energetica e la possibilità di recuperare materia a valle dei processi di raccolta.

Nel tempo discarica e inceneritore si sono rivelate soluzioni integrate - i rifiuti da incenerimento richiedono una discarica di servizio per essere smaltiti e spesso gli inceneritori sono costruiti su terreni prima occupati da discariche - e hanno fondato i loro modelli di business su un ciclo di vita degli impianti medio-lungo (oltre venti anni) e sulla scalarità nel rapporto tra costo di investimento e rendimento energetico (Iaboni e De Stefanis 2017). Il modello di raccolta interdipendente a questi impianti è quello della raccolta stradale, e pur non escludendo la possibilità di avere una parte dei rifiuti raccolti in maniera separata e avviata al riciclo (in specie la frazione secca), l'obiettivo di tali modelli è di generare un volume di rifiuti di almeno il 50-60\% indifferenziato da conferire presso gli impianti di smaltimento. Questo modello, inoltre, richiede automezzi pesanti per essere svolto, un maggior impiego di capitale tecnologico e un minore di lavoro umano, e presuppone una partecipazione poco attiva da parte dell'utenza, che di fatto conferisce quando vuole, senza nessuna forma di controllo o incentivo per i suoi comportamenti.

I sostenitori del modello TMW, hanno messo in evidenza i punti di forza di questo sistema, quando hanno dovuto proteggerlo dagli "attacchi" e dalle delegittimazioni operate dai sostenitori di ZW. In particolare, ricostruendo una parte della letteratura scientifica rivolta alla valutazione critica del modello ZW (Morisi e Paci 2007; Massarutto 2011; Consonni 2018) emergono alcune contro-argomentazioni chiave (Tab.1), tutte coerenti con la visione «linearista» del modello TMW, fondato sui principi della crescita continua, della priorità degli indicatori economici su quelli ambientali, sulla prevalenza del capitale sul lavoro.

Sebbene in questo paragrafo si adotti un metodo comparativo fondato sui reciproci contrasti, bisogna considerare ZW e TMW come i poli opposti di un unico continuum operativo. La gestione dei rifuti è infatti un ambito dove l'azione sociale è fortemente soggetta all'influenza dei contesti locali e dalla path dependence: nulla si crea, e nulla si distrugge partendo da zero o calando nel concreto un modello definito a priori. Osservando il caso italiano, ma si può senz'altro estendere lo sguardo a quasi tutto il contesto dell'Unione Europea e anche oltre (Campos 2013), la gestione dei rifiuti adottata da Comuni e Regioni in base alle rispettive competenze è spesso l'esito di una mediazione tra i due paradigmi. Tuttavia, resta, in prospettiva, che i due modelli tendono ad escludersi reciprocamente se non in maniera assoluta, riducendo l'altro ad una piccola nicchia.

ZW, infatti, si basa sul principio che sia possibile eliminare il concetto stesso di rifiuto, portando al $100 \%$ la raccolta differenziata. Questo obiettivo è ritenuto "utopistico" dai sostenitori del TMW, perché già oggi meno dell' $80 \%$ dei rifiuti urbani può essere credibilmente avviato a riciclo, e una percentuale ancora più bassa può essere trasformato in materia prima secondaria con un mercato di riferimento. Un tempo la principale destinazione delle plastiche riciclate nei paesi europei era la Cina. Da diversi anni, tuttavia, il colosso asiatico ha avviato una politica interna di incremento delle raccolte differenzia. Dal 01 gennaio 2021, sono state bloccate le importazioni di materie prime secondarie. Questo sta mandando in crisi i depositi delle piattaforme di riciclo, che presentano problemi di saturazione, a volte alla base di incidenti e incendi dolosi anche gravi. Inoltre, gli inceneritori di «nuova generazione» avrebbero dei sistemi di filtraggio molto più performanti rispetto ai vecchi impianti, riducendo il rischio per la salute, e presenterebbero delle tecnologie capaci di produrre e stoccare meglio l'energia prodotta dai processi di combustione. Infine, l'estensione dell'IA, oltre a migliorare la fase industriale di trattamento dei rifiuti "a valle",

\footnotetext{
${ }^{2} \mathrm{VR}=(\mathrm{CRD}+\mathrm{CT}-\mathrm{PR})-(\mathrm{CIND}+\mathrm{CS})$ dove VR è il valore di un certo materiale, CRD è il costo della raccolta differenziata, CT il costo delle attività di trattamento finalizzato al recupero a valle della raccolta differenziata, PR il prezzo di mercato del materiale recuperato, CIND il costo della raccolta indifferenziata, CS il costo dello smaltimento indifferenziato.
} 
Tabella 1: Contro-argomentazioni tra TMW e ZW.

\section{TAKE MAKE WASTE MODEL}

Raccolte Differenziata (RD) e filiera riciclo costi troppo elevati e rendimenti troppo bassi per gestire intero volume di rifiuti prodotti dalle città.

$\mathrm{RD}$, anche porta a porta, non elimina completamente gli scarti. Una parte considerevole della $\mathrm{RD}$ in fase di trattamento va sempre in discarica. "Zero Waste» è utopia.

Innovazione tecnologica ha eliminato rischio ambientale legato agli Inceneritori. Lo dimostrano diversi impianti in Europa nel centro delle città (Vienna).

La destinazione delle materie prime secondarie sono mercati ormai saturi come quello cinese. I depositi delle aziende del riciclo sono pieni.

Il consumo di petrolio per la plastica è pari allo $0,5 \%$ del totale, e per carta, vetro e altri RSU, la materia prima estratta è abbondante.

Lautomazione applicata alla RD e agli impianti rende il lavoro sempre più sicuro e cognitivo.

\section{ZERO WASTE MODEL}

Inceneritori hanno un costo di investimento elevatissimo rispetto ai rendimenti. Tutti i business plan TMW si reggono sui contributi pubblici.

Incenerimento congela le RD o impedisce il loro sviluppo perché suo ammortamento si regge su quantità fisse o crescenti di rifiuti da mandare a combustione.

I controlli sui sistemi di filtraggio sono occasionali, gli impianti di incenerimento producono particolato atmosferico necessariamente dannoso per la qualità dell'aria.

La produzione di energia che deriva dall'incenerimento è risibile rispetto a quella impiegata per la gestione dell'impianto.

La lotta alla dispersione della plastica nell'ambiente inizia dal ridurre la quantità di materia prima immessa nel mercato e da un rafforzamento del mercato interno delle materie prime secondarie. Lautomazione produce disoccupazione tecnologica. Applicata ai sistemi di raccolta, può riprodurre la logica del cassonetto indifferenziato.

darebbe nuove opportunità anche per i sistemi di raccolta, che potrebbero tornare su strada, senza i costi elevatissimi del porta a porta, con soluzioni di cassonetti a scomparsa e dotati di sensori per il rilevamento delle utenze conferenti e per il controllo.

Nel modello TMW la raccolta differenziata e le politiche di riduzione dei rifiuti sono considerati strumenti compensativi, ma non esclusivi, di una gestione dei rifiuti efficiente. È significativo che il concetto di ZW sia stato utilizzato, da organizzazioni internazionali come la International Solid Waste Association (ISWA) e dalla Solid Waste Association of North America (SWANA), come un attributo degli impianti di incenerimento, che contribuirebbero a eliminare la frazione residuale non assorbita dalla filiera del riciclo.

I sostenitori del modello ZW invece, non ritengono complementari, ma reciprocamente alternative, le strategie dell'incenerimento e del riciclo. La letteratura scientifica che si è raccolta attorno alle posizioni di ZW (Connett 2013; Dansero et alii 2015; Zaman e Ashan 2019) ha messo in evidenza come gli impianti di incenerimento abbiano un costo di realizzazione così elevato da implicare ammortamenti decennali. Questo rallenterebbe l'adozione di qualsiasi innovazione di breve o medio periodo incompatibile con il modello di business alla base dell'investimento iniziale. Gli inceneritori si reggono sul conferimento di quantità fisse o crescenti di rifiuti, con la conseguenza di bloccare le raccolte differenziate e incentivare un mercato "malato" dove, per esempio, può essere più economico raggiungere un inceneritore fuori regione ma con un costo di smaltimento più basso. Un altro elemento chiave dei processi di delegittimazione di ZW, al netto dei noti aspetti sulle preoccupazioni ambientali e sui rischi per la salute legati alla pratica dell'incenerimento, è quello dell'equilibrio economico dei sistemi incentrati sullo smaltimento e non sul riciclo: gli stessi si reggerebbero infatti o su tariffe elevate e tendenzialmente fisse, o su una quota di contributo pubblico, ad esempio per la produzione di energia, malgrado la stessa sia sempre inferiore a quella consumata per incenerire 3 . Infine, i modelli di raccolta legati al TMW, basandosi essenzialmente sui sistemi stradali e non «porta a porta», ridurrebbero la responsabilizzazione dei cittadini e il ruolo delle reti civiche, riproducendo un sistema individualizzato e mercificato, dove se si paga di più, si è legittimati a inquinare di più.

\footnotetext{
${ }^{3}$ In Italia dal 1992 esiste un incentivo (definito CIP6: Comitato Interministeriale dei Prezzi, provvedimento 6/92) che assimila la produzione di energia da inceneritori a quella derivata da fonti rinnovabili, istituendo un vero e proprio mercato artificiale. Ciò ha consentito per un lungo periodo, per chi gestisce un inceneritore, di vendere l'energia ricavata da processo di combustione a un prezzo circa triplo rispetto a chi produce energia utilizzando fonti convenzionali.
} 
Tabella 2: Principi e roadmapping di «Zero Waste».

\begin{tabular}{|c|c|}
\hline PRINCIPI & 10 Passi verso «Zero Waste» \\
\hline I rifiuti sono risorse & Organizzare sistemi di raccolta differenziata \\
\hline $\begin{array}{l}\text { Un sistema di gestione dei rifiuti è efficiente se si pone il problema di } \\
\text { eliminare completamente gli scarti }\end{array}$ & $\begin{array}{l}\text { Adottare i modelli di raccolta differenziata porta a porta basati } \\
\text { sullesposizione in giorni diversi di diverse tipologie di rifiuto }\end{array}$ \\
\hline $\begin{array}{l}\text { Neanche un chilo di materia organica deve andare in discarica o } \\
\text { incenerimento. }\end{array}$ & $\begin{array}{l}\text { Realizzare impiantistica industriale per il compostaggio e diffondere } \\
\text { compostaggio di comunità/domiciliare. }\end{array}$ \\
\hline $\begin{array}{l}\text { Le filiere verdi possono svilupparsi sul mercato della RD sono foriere } \\
\text { di nuova occupazione }\end{array}$ & $\begin{array}{l}\text { Realizzare piattaforme per il riciclaggio della frazione secca } \\
\text { coinvolgendo imprese locali. }\end{array}$ \\
\hline $\begin{array}{l}\text { La produzione di rifiuti segue tutte le fasi del ciclo di vita dei } \\
\text { prodotti: la logica ZW deve applicarsi sia "a monte" che "a valle" dei } \\
\text { processi di raccolta. }\end{array}$ & $\begin{array}{l}\text { Adottare politiche attive per la riduzione dei rifiuti "a monte": } \\
\text { riducendo le tasse per chi adotta i prodotti alla spina, per chi } \\
\text { produce beni con manutenzione accessibile. }\end{array}$ \\
\hline $\begin{array}{l}\text { lo sviluppo di linee guida per le politiche pubbliche deve orientare il } \\
\text { design di prodotti e servizi (economia circolare) }\end{array}$ & Realizzazione di centri per il riuso e riparazione dei beni durevoli. \\
\hline $\begin{array}{l}\text { Paga di più chi inquina di più, ma nessuno ha diritto a inquinare più } \\
\text { di quanto dovrebbe solo perché paga. }\end{array}$ & $\begin{array}{l}\text { Adozione dei sistemi di tariffazione puntuale basato sul principio } \\
\text { "pay as you throw». }\end{array}$ \\
\hline L'innovazione tecnologica va rivolta alla bonifica delle discariche. & $\begin{array}{l}\text { Realizzazione di impianti per il recupero dei rifiuti non riciclabili, } \\
\text { speciali e industriali }\end{array}$ \\
\hline $\begin{array}{l}\text { Il ciclo di vita dei prodotti deve essere più lungo e va abolita } \\
\text { l'obsolescenza programmata }\end{array}$ & $\begin{array}{l}\text { Insediamento di centri di ricerca e riprogettazione di beni secondo il } \\
\text { modello dell'economia circolare }\end{array}$ \\
\hline $\begin{array}{l}\text { «Zero Waste» è possibile e il percorso va definito attraverso un piano } \\
\text { con orizzonti temporali definiti. }\end{array}$ & Raggiungimento entro un certo termine dell'azzeramento dei rifiuti. \\
\hline
\end{tabular}

Nei lavori di Curran e Williams (2012) e di Leo e Salvia (2017) è possibile ricavare i principi su cui si basa il modello ZW (Tabella 2) e, grazie al lavoro di divulgazione e sistematizzazione condotto da Connett (2013) e da Ercolini (2018), tali principi si sono tradotti anche in un roadmapping costituito da dieci step, dove è possibile verificare la correlazione tra apparato simbolico e materiale di ZW, e che viene messo a disposizione di amministrazioni, aziende e comunità che vogliono aderire al modello.

Sia i principi, sia il roadmapping, inquadrano un sistema di valori e pratiche attraverso cui convertire l'economia dei rifiuti di un territorio.

Dal punto di vista dei valori, ZW non compie particolari innovazioni: ripercorre e applica, in un settore nevralgico della vita urbana, i principi dell'ecologia industriale, della bioeconomia, del metabolismo urbano (Zaman e Ashan 2019). Alla base di ZW vi è una critica della visione lineare dello sviluppo economico e dei suoi indicatori di performance. La gestione dei rifiuti è ricodificata sia rispetto alle metriche del valore e ai ruoli che si attribuiscono agli attori sociali: priorità agli obiettivi ambientali rispetto a quelli di remunerazione del capitale investito; più autonomia e potere ai municipi rispetto agli organi centrali; maggiore coinvolgimento delle reti associative e civiche nella co-progettazione dei servizi, nella comunicazione e nel controllo sulla gestione; centralità delle piccole e medie imprese legate al territorio, rispetto alla GDO, alle grandi imprese de-territorializzate.

Dal punto di vista delle infrastrutture materiali e dei dispositivi organizzativi, ZW si distingue per alcune soluzioni innovative ed esclusive. La prima è la raccolta differenziata «porta a porta», che propone di adottare in tutti i contesti, compresi quelli metropolitani, e che ha come conseguenza lo sviluppo di un nuovo design per automezzi di raccolta, dispositivi per l'esposizione del rifiuto, isole ecologiche, impianti di trattamento. Poi il riferimento al compostaggio domestico e di comunità, come soluzione sistematica, da adottare in tutti i luoghi, compresi quelli urbani, che riguarda una percentuale di rifiuto pari. L'innovazione digitale è pensata per l'implementazione di sistemi di tariffazione che si basano sul riconoscimento dell'utente, in modo da disincentivare l'esposizione di rifiuto indifferenziato. Anche sull'impiantistica, ZW propone delle soluzioni diverse rispetto al modello TMW, ad esempio, ostacolando non solo inceneritori e discariche, ma anche la realizzazione di mega-impianti per la RD o di compostiere di comunità elettrificate.

Ma chi ha elaborato queste idee e innovazioni pratiche? Come le ha diffuse, in particolare, in Italia? Il prossimo paragrafo, costruito sulla base degli esiti delle interviste con gli osservatori privilegiati, descrive una parte dei 
"luoghi" del discorso di ZW, cioè gli eventi, gli attori e le piattaforme collaborative che hanno generato il suo sviluppo in Italia.

\section{I LUOGHI DEL DISCORSO}

ZW è un fenomeno internazionale, la cui genesi ufficiale è collocabile nel 2002, quando viene costituita la Zero Waste International Alliance (ZWIA) a seguito di un ciclo di conferenze organizzate da scienziati e attivisti di associazioni ambientaliste e grassroots impegnati nell'affermare una gestione dei rifiuti senza inceneritori.

ZW in Italia ha un radicamento importante, testimoniato dalla presenza di alcune strutture associative che promuovono la strategia a livello nazionale. Una delle più importanti è il Comitato di Garanzia Nazionale costituito da Zero Waste Europe e Italia, in collaborazione con l'osservatorio del Comune di Capannori. Fanno parte della rete circa 315 Comuni, con una popolazione complessiva di circa 7 milioni di abitanti serviti. Si tratta di amministrazioni locali che hanno approvato atti di adesione formale, come delibere di consiglio comunale o giunta, dove prendono l'impegno di attuare compiutamente la strategia.

In Italia la diffusione di ZW è stata facilitata dalle frequenti emergenze rifiuti che si sono verificate nelle principali città italiane (Milano, Napoli, Roma), con strade e piazze trasformate per giorni in discariche. L'analisi delle cause e delle contraddizioni politiche dietro l'origine di tali fenomeni ha contribuito infatti a mostrare con evidenza i limiti economici e ambientali del modello TMW e a dare spazio all'"eresia" di ZW.

La crisi dei rifiuti nella città di Milano del 1995, potrebbe essere considerato l'evento-culla di ZW in Italia. Nel 1995 Milano era servita dalla discarica di Cerro Maggiore, dove conferiva quotidianamente il 97\% dei propri rifiuti. A ottobre del 1995, i cittadini di Cerro organizzarono dei blocchi stradali, perché la Regione Lombardia decise di prorogare per altri due anni il ciclo di vita della discarica, chiusa per saturazione e problemi ambientali. Si aprì una crisi istituzionale che ebbe come effetto il blocco della raccolta dei rifiuti in tutta l'area metropolitana di Milano, dove per le strade restarono fino a trentamila tonnellate di immondizia (Ganapini 2004). Dopo aver raggiunto un accordo con la Regione Emilia-Romagna per rimuovere i rifiuti dalle strade, l'azienda pubblica milanese per i rifiuti (AMSA) organizzò in un sito industriale dismesso di proprietà della Maserati, un impianto di trattamento meccanico biologico $(\mathrm{TMB})^{4}$, dalla capacità di circa 2.000 tonnellate/giorno. Parallelamente, il Comune avviò la raccolta differenziata porta a porta integrale, compresa la frazione organica, prima città in Europa per dimensioni a adottare un sistema così radicale. Milano adottò nella propria realtà urbanistica, sistemi già sperimentati in Germania e Olanda, ma su scale molto più ridotte. Per la prima volta furono sperimentati automezzi di piccola cilindrata, che avevano pertanto una portabilità inferiore ai modelli con tre-quattro assi delle raccolte stradali, ma una maggiore capacità di raggiungimento e mobilità nei contesti metropolitani milanesi. Il piano realizzato da Milano fu integrato dall'apertura di due «riciclerie», isole ecologiche deputate al recupero di rifiuti ingombranti, a cui si ispireranno i Centri per il Riuso promossi dal movimento ZW. Fondamentale per la riuscita dell'esperienza fu infine la costituzione di un Forum civico, guidato da Guido Viale, che raccolse la società civile meneghina in un progetto condiviso di sensibilizzazione della cittadinanza.

Un secondo evento chiave fu l'emergenza rifiuti a Napoli. È nota a livello internazionale la drammatica e interminabile crisi ecologica che ha colpito il capoluogo campano, tra il 2008 e il 2011, con migliaia di tonnellate di rifiuti lasciate a terra per settimane, roghi e commissariamenti degli enti locali per inerzia o infiltrazione mafiosa. Un evento che si è legato, non solo simbolicamente, al tema delle discariche di rifiuti industriali nella cosiddetta «Terra dei fuochi», tra la provincia di Napoli e Caserta, con centinaia di ettari di terreni agricoli resi inservibili

\footnotetext{
${ }^{4}$ Nella gestione dei rifiuti il trattamento meccanico-biologico (TMB) è una tecnologia di trattamento a freddo dei rifiuti indifferenziati (e/o residuali dopo la raccolta differenziata) che sfrutta l'abbinamento di processi meccanici a processi biologici quali la digestione anaerobica e il compostaggio. Appositi macchinari separano la frazione umida (l'organico da bioessicare) dalla frazione secca (carta, plastica, vetro, inerti, ecc.); quest'ultima frazione può essere in parte riciclata oppure usata per produrre combustibile derivato dai rifiuti (CDR) rimuovendo i materiali incombustibili.
} 
dal sotterramento illegale di scarti industriali, spesso provenienti da industrie del nord Italia. Nel 2009, nel pieno dell'emergenza, grassroots radicati nel territorio campano in contatto col network nazionale ZW, si mobilitarono e organizzarono una tre giorni di dibattiti e incontri con le amministrazioni locali. L'obiettivo fu di mettere in contatto esperti e case studies di ZW con i Sindaci e i Consiglieri comunali delle città campane colpite dall'emergenza. $\mathrm{Ne}$ nacque un documento, «La Carta di Napoli», dove furono individuate le caratteristiche essenziali per poter definire una comunità a «rifiuti zero». La Carta ebbe la finalità di stabilire i confini identitari del movimento ZW, e di sollevare l'attenzione dei vari livelli governativi impegnati nella risoluzione della crisi rifiuti a Napoli. Non è un caso che, qualche anno dopo, sarà chiamato a gestire l'azienda municipalizzata locale Raphael Rossi, tra le figure più rappresentative del movimento ZW in Italia.

Milano e Napoli hanno costituito due grandi occasioni per mostrare le debolezze del modello TMW e le opportunità di quello ZW. Tuttavia, sono state significative anche perché hanno fatto da incubatrici per l'expertise che, nell'immediato futuro, avrebbe consolidato e diffuso ZW in tante altre località italiane.

Uno dei "luoghi" dove è maturata la pratica discorsiva di ZW è la Scuola Agraria del Parco di Monza, un ente pubblico regionale che si occupa di verde ornamentale, ma che ha proprio interno ricercatori esperti nel compostaggio che, in tempi in cui sembrava assurdo pensarlo, compresero quanto fosse cruciale separare la frazione organica da quella secca anche nei contesti urbani.

La Scuola progettò nel 1993 uno dei primi sistemi di raccolta differenziata porta a porta, nel piccolissimo Comune di Bellusco, a pochi chilometri da Milano. I suoi tecnici furono chiamati a gestire l'emergenza-Milano. La Scuola, sull'onda del successo di Bellusco e Milano, organizzò mensilmente incontri e seminari presso la propria sede, coinvolgendo chi cominciava a praticare dal basso le esperienze ZW, anche prima che fossero così definite. Fu inoltre chiamata a redigere i piani regionali e provinciali di decine di amministrazioni in Italia, ben oltre i confini della Lombardia. Si formava così una comunità di pratiche che univa approccio scientifico e applicazione concreta nei territori, ed elaborava una vera e propria "eresia" nella gestione dei rifiuti. Grazie a queste esperienze, e alle prime pubblicazioni in cui le pratiche di ZW vengono sistematizzate (Tornavacca e Onida 1999), nacque, dalla seconda metà degli anni Novanta e, in misura maggiore, a partire dal nuovo millennio, un intenso dialogo tra attori politici locali ed expertise di ZW.

Altri due luoghi di elaborazione del discorso ZW in Italia furono, indubbiamente, la Provincia di Treviso, e in particolare l'azienda pubblica Contarina Spa, e il Comune di Capannori, con la sua azienda Ascit. Ancora oggi sono le "madonne pellegrine" di ZW, i casi di successo che vengono mostrati (con visite aziendali, organizzazione di seminari a livello locale) ad amministratori locali, associazioni e comitati cittadini, per far sì che sviluppino quei modelli socio-tecnici nei propri contesti territoriali.

Contarina è un'azienda a totale capitale pubblico, che opera su un territorio di circa 50 municipalità, riunendo quasi interamente la Provincia di Treviso, nel Veneto, servendo circa 554mila abitanti, con un fatturato, nel 2018, di quasi 80 milioni di euro e un utile netto di 1,2 milioni di euro. Nel 2018 Contarina ha raggiunto una media di riciclo del 85,6\%, nonché uno dei quantitativi pro-capite di produzione del rifiuto più bassi d'Europa, con $58 \mathrm{~kg}$ a persona, quando la media Italiana è $236 \mathrm{~kg}$, e quella Europea supera i $450 \mathrm{Kg}$ all'anno (Minoja e Romano 2020). Contarina ha introdotto la raccolta differenziata porta a porta nel 1997, in un territorio dove veniva eseguita la raccolta «stradale indifferenziata». Per facilitare la separazione del rifiuto umido, Contarina fornì alle utenze un mastello forellato di dimensioni inferiori al bidone in cui il rifiuto doveva essere inserito per l'esposizione stradale. I tecnici di Contarina notarono infatti che vi era una correlazione tra la quantità di rifiuti prodotta e il volume del bidone utilizzato per raccoglierla: più offerta di spazio, equivaleva ad una domanda maggiore. Tra le altre innovazioni introdotte da Contarina, e diventate un modello organizzativo per molte altre realtà italiane, vi è la tariffazione puntuale, in cui parte della tassa sui rifiuti è pagata in base al numero dei conferimenti del rifiuto non riciclabile.

Ascit è la società di gestione del Comune di Capannori, circa 45 mila abitanti, e di altri Comuni del comprensorio lucchese. Qui, nel 1994, la Regione Toscana aveva deciso di insediare un inceneritore. Il progetto verrà tuttavia bloccato da una straordinaria mobilitazione della società civile locale. Il Comune di Capannori fu tra i primi ad aderire alla rete internazionale «Zero Waste», adottando in Consiglio comunale una delibera con l'impegno di 
implementare i «dieci passi» stabiliti dai teorici di «Zero Waste». Capannori attuò la tariffazione puntuale, al pari di Contarina e altre esperienze italiane, ma introdusse anche nuove iniziative, tra cui un Regolamento per la tassa sui rifiuti in cui vennero premiati gli esercenti che adottavano contenitori alla spina, o che introducevano piccole innovazioni nel packaging dei prodotti, per evitare la produzione di imballaggi. Il Comune inoltre si impegnò a sensibilizzare grandi imprese, come Lavazza, a produrre capsule per il caffè con materiali riciclabili, e a diffondere l'uso dei pannolini lavabili, detassando le famiglie che li acquistavano. Sempre a Capannori, vennero inaugurati due centri del riuso, sulla falsariga dell'esperienza milanese, dove i rifiuti ingombranti venivano conferiti e rilavorati per recuperare le materie riutilizzabili. Tuttavia, l'elemento forse più distintivo dell'esperienza di Capannori, fu la sensibilità verso la produzione di conoscenza condivisa sul tema ZW e la creazione di una rete extra-territoriale, di partenariato con altri Comuni, aziende pubbliche e private interessate a entrare nella comunità ZW italiana, di cui è esemplare la costituzione dell'Osservatorio Nazionale Rifiuti Zero.

L'Osservatorio ha istituito un centro di ricerca sulla gestione dei rifiuti. Il focus è sulla composizione del secco residuo e delle frazioni non riciclabili, per riorientare il design produttivo, e per sensibilizzare famiglie e aziende, sulla necessità di adottare comportamenti coerenti con una visione circolare dell'economia. L'Osservatorio è inoltre il punto di riferimento per il network ZW italiano, a cui hanno aderito, ad oggi, circa 300 amministrazioni locali, le quali si riuniscono annualmente per condividere formazione e conoscenza condivisa nelle proprie pratiche locali.

\section{GESTIONE DEI RIFIUTI E TRASFORMAZIONI SOCIALI}

La ricostruzione degli eventi che hanno portato al radicamento di ZW in Italia è utile anche per mettere a fuoco alcuni processi di mutamento sociale che proprio in ZW trovano traccia e causa. Cambiare il modo di concepire e gestire il ciclo dei rifiuti ha infatti delle implicazioni rilevanti sulla struttura e l'organizzazione delle comunità urbane.

Un primo livello dove osservare tali trasformazioni è quello dei rapporti di potere che si stabiliscono tra gli attori protagonisti dei processi di innovazione socio-tecnica. Lo schema tradizionale del trasferimento tecnologico (Van de Ven 2000; Rogers 2003; Ramella 2015; Marciano et alii 2020) vede una divisione del lavoro tra gli attori dei sistemi di innovazione per cui l'azienda privata esprime i bisogni, le Università o i centri di ricerca rispondono attraverso soluzioni puntuali, gli istituti di credito finanziano le innovazioni e ne ricavano profitti o equities. È il modello della tripla elica (Etzkowitz e Leydesdorff 2000), che comprende diverse varianti sullo stesso tema: ad esempio, che la spinta iniziale al processo innovativo avvenga in ambito di R\&S - come conseguenza di opportunità offerte dall'applicazione di nuove tecnologie e conoscenze - e sia intercettato da strutture di venture capital e poi offerto ad aziende o incorporato in nuove aziende (il «modello Silicon Valley», cfr. Ferrary e Granovetter 2009). Sono gli stessi criteri su cui è costruito il modello TMW: anche in questo modello i rifiuti non sono un «problema» ma una «risorsa», che va tuttavia capitalizzata negli impianti. Le principali eccedenze si verificano nell'attività di smaltimento che sono capital intensive, e arrivano in alcuni modelli applicativi, specie quelli dove discariche e inceneritori non sono molti, a rappresentare oltre il 50\% del valore aggiunto di tutto il ciclo dei rifiuti.

ZW è un campo di inversione di tale tendenza. Il trasferimento tecnologico e di conoscenza avviene in una struttura di incubazione completamente diversa. Ad esprimere i bisogni sono enti locali e gli attori collettivi della società civile locale; a sviluppare soluzioni reti collaborative tra ricercatori, manager e tecnici di aziende pubbliche. La finalità della ricerca è di offrire soluzioni puntuali e non scalari, adeguate a singoli contesti, e non a mercati generalisti. Il meccanismo è oliato dall'esigenza di contenere rischi ambientali (ad esempio la realizzazione di un inceneritore o la saturazione di una discarica), di alimentare un'economia locale e job intensive che sposti il valore aggiunto dal capitale al lavoro. La diffusione delle innovazioni avviene in ambiti informali, di knowledge management «tacito» (Nonaka 1991), senza brevetti o modelli di gestione istituzionalizzati. ZW alimenta pertanto un settore di innovazione marginale (Barbera e Jones 2020), dove la creazione di prodotti e processi migliorativi per l'organizzazione che li adotta è una qualità diffusa dell'azione sociale, e non si riconosce nella visione individualizzata, del «genio singolo» e della start up dal potenziale remunerativo clamoroso. 
Un secondo livello dove osservare le trasformazioni sociali implicite in questa esperienza è, direttamente collegato al primo, quello dei processi di rimunicipalizzazione e di ritorno alla gestione pubblica dei servizi a rilevanza economica locale. La liberalizzazione di tali servizi e la conversione dei loro sistemi di gestione secondo le strategie organizzative delle aziende private è stato uno dei pilastri del new public management (Lane 2002), attraverso cui si è promosso quello che Harvey (2011) ha definito lo shift tra «governance urbana imprenditoriale e manageriale», tra un attore pubblico direttamente investito nella gestione dei processi industriali relativi ai servizi di cui è titolare, a uno di facilitatore e regolatore. Sebbene ZW non abbia una posizione esplicita sulla ownership dei servizi di raccolta, e anzi diverse sue strutture di riferimento partecipino in veste di progettisti di sistemi di raccolta e trattamento dei rifiuti gestiti da soggetti privati, è altresì evidente che tutti gli esempi più rilevanti e di successo della strategia in Italia siano stati adottati da aziende a totale capitale pubblico (Romano et alii 2019), e che si sia stabilito un forte legame tra le argomentazioni care a ZW, in particolare quelle sul ruolo da attribuire ai movimenti civici e alle piccole imprese del territorio nella cura dei commons, e le rivendicazioni che hanno dato vita al movimento per i beni comuni. ZW è quindi diventato, in alcuni casi di rimunicipalizzazione, il contenuto fondamentale che enti locali e movimenti sociali hanno utilizzato per giustificare tale scelta rispetto a quella più consolidata della privatizzazione: lo dimostrano decine di casi di reinternalizzazione dei servizi di igiene urbana in corso, come quelli in Provincia di Forlì, Latina, Roma, Nuoro, Barletta, Reggio Calabria, Belluno (Minoja e Romano 2021), che hanno legato la scelta pubblica alla necessità di realizzare un programma a «rifiuti zero» incompatibile con la presenza di capitali privati rilevanti.

Un terzo livello di osservazione delle trasformazioni sociali è quello relativo alla penetrazione di pratiche discorsive come ZW nell'arena delle policy europee e nazionali. L'Unione Europea riconosce Zero Waste Europe come uno stakeholder di primo livello nell'articolazione delle politiche sull'economia circolare e sull'ambiente. Ricercatori e professionisti che aderiscono a ZW sono parte integrante dei tavoli dove vengono prodotte e discusse le politiche europee, e i risultati di questa influenza sono evidenti: nel prossimo "pacchetto" sulla circular economy l'energia prodotta tramite valorizzazione energetica non sarà più riconosciuta come indicatore positivo di performance; la frazione organica è oggetto di direttive sempre più restrittive sul suo conferimento in discarica o incenerimento; le raccolte differenziate porta a porta e sul modello PAYT («pay as you throw», tariffazione puntuale) sono considerate la prima opzione per le città. I regolamenti e gli standard per l'autorizzazione di impianti di incenerimento e discariche diventano sempre più esigenti. Anche uno sguardo al Piano di Rinascita e Resilienza (PNRR), e in particolare il capitolo dedicato alla green economy, è fortemente influenzato dalle soluzioni proposte da ZW, sul compostaggio di comunità, sulla realizzazione di impianti per il trattamento dei rifiuti riciclati o per la digestione anaerobica con produzione di biogas.

Questo non vuol dire che non vi siano iniziative normative contraddittorie, né che i sostenitori di tesi avverse a ZW non abbiano ascolto presso le istituzioni politiche regolatorie: solo che non sono più l'unica voce, non sono più l'unico punto di vista espresso dalle istituzioni. È questo il segno che vi è un processo di contro-egemonia culturale in corso, e le aspirazioni e i progetti di un'élite - spesso legata al mondo accademico e dei movimenti sociali più che a quello della ricerca e sviluppo industriale o finanziaria - divengono modello per l'intera società.

\section{CONCLUSIONI}

L'articolo ha ricostruito la genesi e le caratteristiche del fenomeno ZW in Italia, proponendo una sua interpretazione attraverso il concetto di pratica discorsiva, che consente di osservare le interdipendenze tra aspetti simbolici e materiali. A questo intento si è aggiunto quello di avviare una riflessione, ancora poco radicata negli studi sociologici, sulle implicazioni che la gestione dei rifiuti ha per i processi di mutamento sociale.

Lo studio esposto ha diverse vulnerabilità, tra cui quella di non offrire uno spaccato internazionale alla lettura di un fenomeno che invece si pone come soluzione globale ed è fortemente radicato anche in altre realtà. I sistemi di raccolta e trattamento dei rifuti incorporano infatti, nei propri dispositivi tecnologici e organizzativa, una precisa visione dei rapporti sociali e dei valori culturali, che potrebbe beneficiare di uno sguardo comparativo non solo 
tra Italia e i paesi limitrofi, ma oltre l'Europa, dove ZW è fortemente radicato, ad esempio Australia, Nuova Zelanda ed East Coast statunitense.

Lo studio, nel presentare la genesi e il radicamento di ZW, non ha potuto osservare i casi di insuccesso né i tanti problemi applicativi affrontati dalle organizzazioni impegnate nella sua diffusione sul territorio, tra cui alcune descritte dalle interviste con gli esperti sono la crescita dei costi di raccolta, l'aumento dei conferimenti impropri, frammentazione gestionale, l'incoerenza tra impianti di trattamento disponibili e strategie di raccolta, le difficoltà normative legate all'implementazione di compostaggio e tariffazione puntuale. Questo, del resto, non ha attinenza con l'oggetto della ricerca qui presentata, che non è l'efficacia di ZW rispetto ad altri modelli, ma il modo in cui il suo apparato socio-tecnico si è affermato nella realtà italiana.

Tra gli aspetti poco approfonditi, ma forse utili ad uno sviluppo successivo, vi è quello della potenzialità di un lavoro di squadra tra scienze sociali, umane e tecnologiche. Le amministrazioni regionali e comunali, oltre che le aziende di gestione dei servizi, hanno bisogno di redigere piani strategici facendo adeguate analisi di sfondo e dei bisogni, migliorando la qualità e la complessità delle attività di comunicazione con i cittadini. Inoltre, hanno bisogno di aprire al contributo dei sociologi anche le fasi di progettazione "hard", quelle dove si decidono mezzi, personale e strumenti con cui si effettuano i servizi, oggi preda di automatismi e di soluzionismi astratti, come se le scelte industriali fossero governate da una logica a priori rispetto ai bisogni e alle possibilità peculiari espresse dai singoli territori.

\section{BIBLIOGRAFIA}

Avallone G. (2014), Terra di conflitti. Rifiuti, espropriazione e movimenti socio-ecologici in Campania, in «Prisma. Economia Società Lavoro», 3: 78-92.

Barbera F., Jones I.R. (2020, eds.), The Foundational Economy and Citizenship: Comparative Perspective on Civil Repair, Bristol: Bristol University Press.

Barbera F., Parisi T. (2018), Innovatori sociali. La sindrome di Prometeo nell'Italia che cambia, Bologna: il Mulino.

Bacchi C., Bonham J. (2014), Reclaiming discursive practices as an analytic focus: Political implications, in «Foucault Studies», 17: 173-192.

Campos M. (2013), The function of waste urban infrastructures as heterotopias of the city: Narratives from Gothenburg and Managua, in M. Campos e C. Hall, Organising waste in the city: International perspectives on narratives and practices, Bristol/Chicago: Bristol University Press.

Cianciullo A., Fontana E. (2005), Dark economy. La mafia dei veleni, Torino: Einaudi.

Clifton J., Warner M.E., Gradus R., Bel G. (2019), Re-municipalization of public services: trend or hype?, in «Journal of Economic Policy Reform», 24, 3: 293-304.

Connett P. (2013), The zero waste solution, Chelsea: Chelsea Green Publishing.

Crivello S. (2016), Capitale, natura e città: ecologia politica urbana dell'inceneritore del Gerbido a Torino, in «Sociologia urbana e rurale», 109: 22-39.

Curran T., Williams I.D. (2012), A zero waste vision for industrial networks in Europe, in «Journal of Hazardous Materials», 207-208: 3-7.

Consonni D. (2018), Cosi la scienza incenerisce i “no termo", in «Il Foglio.it», 22 dicembre 2018, https://www.ilfoglio.it/granmilano/2018/11/22/news/termovalorizzatori-scontro-lega-m5s-differenziata-scienza-spiega-datilombardia-225784/

Dansero E., Putilli M., Tacco N. (2015). Geopolitiche dei rifiuti. Attori, scale e processi decisionali nella localizzazione di due inceneritori in Provincia di Torino in «Bollettino della società geografica italiana», 8: 469-490.

Ercolini R. (2018), Rifuti Zero. Dieci passi per la rivoluzione ecologica dal premio Nobel per l'ambiente, Milano: Baldini e Castoldi.

Etzkowitz H., Leydesdorff L. (2000), The dynamics of innovation: from National Systems and "Mode 2" to a Triple Helix of university-industry-government relations, in «Research policy», 29, 2: 109-123. 
Ferrary M., Granovetter M. (2009), The role of Venture Capital Firms in Silicon Valley>s Complex innovation network, in «Economy and Society», 38, 2: 326-359.

Ganapini W. (2004), Ambiente made in Italy, Reggio Emilia: Aliberti Editore.

Harvey D. (2011), Le città ribelli. I movimenti urbani dalla Comune di Parigi a Occupy Wall Street, Milano: Il Saggiatore.

Iaboni V., De Stefanis P. (2007), Aspetti economici del recupero energetico, Roma: Enea.

Lane J.E. (2002), New Public Management: An Introduction. London: Routeldge.

Leo S., Salvia M. (2017), Local strategies and action plan towards resource efficiency in South East Europe, in «Renewable Sustainable Energy Review », 68: 286-305.

Magnani N. (2011), Attori sociali e fattori materiali nei conflitti ambientali. Il caso dell'inceneritore di Trento, in L. Pellizzoni, Conflitti ambientali. Esperti, politica, istituzioni nelle controversie ambientali, Bologna: il Mulino.

Marciano C. (2017), Economia circolare. Critica di un paradigma emergente nella policy ambientale dell'UE, in «Prisma. Economia Società Lavoro», 1-2: 14-25.

Marciano C., Cassone Idone V., Onnis E., Paini G. (2020), Università e territorio. Il modello del Knowledge Interchange e il caso Piemonte 2029, in «Autonomie Locali e Servizi Sociali», 1: 107-127.

Martone V. (2012), I conflitti ambientali come sperimentazione di comunità di rischio territorializzato, in «Sociologia Urbana e Rurale», 97: 67-84.

Martone V. (2016), La governance dei rifiuti urbani: la progettazione partecipata della raccolta differenziata a Scampia, in G. Punziano, Società Economia e Spazio a Napoli. Esplorazioni e riflessioni, GSSI Social Sciences n. 28.

Massarutto A. (2011), Anche la green economy ha una parte marrone. La gestione dei rifiuti e l'utopia "rifuti zero", in «'Industria», 2: 245-264.

Mattei U. (2018), Diritto, capitale e limiti, in «Parolechiave», 1: 115-126.

Mazzarella F., Escobar-Tello C., Mitchell V. (2016), Moving textile artisans> communities towards a sustainable future: a theoretical framework, in AA.VV., Proceedings of 50th Anniversary Design Research Society Conference, Brighton, UK, 27-30 giugno 2016.

Mazzette, A., Sgroi, E., (2007), La metropoli consumata. Antropologie, architetture, politiche, cittadinanze. Milano: Franco Angeli.

Minervini D. (2016), Strategie pubbliche e aziendalizzazione dei servizi di gestione dei rifuti urbani, in «Sociologia del lavoro», 142: 29-48

Minoja M., Romano G. (2021), Managing intellectual capital for sustainability: Evidence from a re-municipalized, publicly owned waste management firm, in «Journal of Cleaner Production», 279: 123213.

Morisi M., Paci A. (2007, cur.), Il bisogno di decidere. Termovalorizzatori: dalla politica del rifuto al rifuto della politica, Bologna: il Mulino.

Nonaka I. (1991), The knowledge creating company, in: «Harvard Business Review», 69: 96-104.

Osti G. (2002), Il coinvolgimento dei cittadini nella gestione dei rifuti, Milano: FrancoAngeli.

Pellegrino V. (2011), Conflitti ambientali e nuovi soggetti politici. Riflessioni sulle rivolte 'eco-epidemiologiche', in «La società degli individui», 42: 81-92.

Pellizzoni L. (2011), Conflitti ambientali. Esperti, politica, istituzioni nelle controversie ecologiche, Bologna: il Mulino.

Pietzsch N., Duarte Ribeiro J. L., de Medeiros J. G. (2017), Benefits, challenges and critical factors of success for Zero Waste: A systematic literature review, in «Waste Management», 67: 324-353.

Ramella F. (2015), Sociologia dell'Innovazione Economica. Bologna: il Mulino.

Rodić L., Wilson D.C. (2017), Resolving Governance Issues to Achieve Priority Sustainable Development Goals Related to Solid Waste Management in Developing Countries, in «Sustainability», 9: 404 https://doi.org/10.3390/ su9030404

Rogers E. (2003), Diffusion of innovations, New York: Free Press

Romano G., Ferreira D.C., Marques R.C., Carosi L. (2020), Waste services' performance assessment: The case of Tuscany, Italy, in «Waste Management», in press 
Romano G., Marciano C., Fiorelli M.S. (2021, eds.), Best practices in Urban Solid Waste Management: Ownership, Governance, and Drivers of Performance in a Zero Waste Framework, London: Emerald Limited Series.

Strassoldo R. (1993), Le radici dell'erba. Sociologia dei movimenti ambientali di base, Napoli: Liguori Editore.

Tipaldo G. (2013), Le due torri. Scienza e Politica nel caso dell'inceneritore di Torino, in V. Pellegrino, La Scienza Incerta e la Partecipazione, Trieste: Scienze Express Frontiere.

Tornavacca A., Onida M. (1999), Da rifiuti a risorse, Mestre: Ecoistituto del Veneto.

Utilitalia (2020), Green Book: i dati sulla gestione dei rifuti urbani in Italia. Roma: Pubblimedia srl.

Van de Ven A., Polley D., Garud R., Venkataraman S. (2000), The innovation journey, New York: Oxford University Press.

Zaman A., Ashan T. (2019), Reconsidering waste management for the future, London: Routledge.

Zaccaria A.M., Ragozini G., Piscitelli A. (2012), Il caso della differenziata porta a porta a Napoli. risultati della ricerca, in S. Consiglio, G. Ragozini e A.M. Zaccaria, Soddisfazione del cittadino e politiche pubbliche. La raccolta differenziata a Napoli, Roma: Carocci Editore. 